\title{
Two populations among the metal-poor field RR Lyrae stars
}

\author{
T. V. Borkova and V. A. Marsakov
}

\author{
Space Research Department at Rostov State University, and Issac Newton Institute of Chili Rostov-on-Don Branch Stachki 194, \\ Rostov-on-Don 344090, Russia \\ e-mail: borkova@rsusu1.rnd.runnet.ru; marsakov@ip.rsu.ru
}

Received 17 April 2002 / Accepted 17 September 2002

\begin{abstract}
We compute the spatial velocity components and the galactic orbital elements for 209 metal-poor $([\mathrm{Fe} / \mathrm{H}]<-1.0)$ RRLyrae (ab) variable stars in the solar neighborhood using proper motions, radial velocities, and photometric distances available in the literature. The computed orbital elements and published heavy element abundances are used to study relationships between the chemical, spatial, and kinematical characteristics of nearby field RR Lyrae variables. We observe abrupt changes in the stellar spatial and kinematical characteristics when the peculiar velocities relative to the local standard of rest cross the threshold value, $V_{\mathrm{pec}} \approx 280 \mathrm{~km} \mathrm{~s}^{-1}$. This provides evidence that the general population of metal-poor RRLyrae stars is not uniform, and includes two spherical subsystems occupying different volumes in the Galaxy. Based on the agreement between typical parameters of corresponding subsystems of field RRLyrae stars and of the globular clusters, studied by us earlier, we conclude that metal-poor stars and globular clusters can be subdivided into two populations, but using different criteria for stars and clusters. We suppose that field stars with velocities below the threshold value and clusters with extremely blue horizontal branches form the spherical, slowly rotating subsystem of the proto-disk halo (related by its origin to the Galactic thick disk). It has a negligible, but non-zero, vertical metallicity gradient. Field stars with fast motion and clusters with redder horizontal branches constitute the spheroidal subsystem of the accreted outer halo, which is approximately two times larger in size than the first subsystem. It has absolutely no metallicity gradients, most of its stars have eccentric orbits, many stars display retrograde motion in the Galaxy, and their ages are comparatively low, supporting the hypothesis that the objects in this subsystem have an extragalactic origin.
\end{abstract}

Key words. stars: variables: RRLyr - Galaxy: kinematics and dynamics - Galaxy: stellar content - Galaxy: halo

\section{Introduction}

The presence of two different populations with separate histories in the metal-poor halo was suggested by Hartwick (1987). He showed that model of the dynamics of RR Lyrae variables with metallicities $[\mathrm{Fe} / \mathrm{H}]<-1.0$ required two components: one spherical and a somewhat flattened component that is dominant at galactocentric distances less than the radius of the solar circle. The idea that there are two subsystems in the metal-poor halo has already been addressed in several investigations focused on globular clusters. It turned out that globular clusters present a distinctive inner feature (the morphology of their horizontal branches), which makes it possible to distinguish the individual metal-poor clusters in terms of different halo subsystems. It was found that halo clusters that have redder horizontal branches for a given metallicity (i.e. with horizontal branches showing a considerable number of stars on the lowtemperature side of the Schwarzschild gap), are predominantly outside the solar circle. Furthermore, they exhibit larger velocity dispersion, slower circular velocity (a significant number having retrograde orbits), and are, on average, younger than clusters with extremely blue horizontal branches, which are

Send offprint requests to: V. Marsakov, e-mail: marsakov@ip.rsu.ru concentrated within the solar circle (Da Costa \& Armandroff 1995; Borkova \& Marsakov 2000). Note that relative ages for globular clusters were determined by different authors with the help of observed high-precision colour-magnitude diagrams and theoretical isochrones. Therefore, these ages are independent of horizontal branch morphology, and the relative youth of some metal-poor globular clusters is beyond doubt (see compilative catalogue of homogeneous age dating of 63 globular clusters by Borkova $\&$ Marsakov 2000). The explanation suggested for the difference between these two populations was that the subsystem of clusters with extremely blue horizontal branches (i.e. the older halo) formed together with the Galaxy as a whole, whereas the clusters of the younger halo subsystem formed from fragments captured by the Galaxy from intergalactic space at later stages of its evolution (Zinn 1993). Recent observations (Ivezic et al. 2000; Vivas et al. 2001) suggest strong evidence in favor of the hypothesis that star and also globular clusters of the outer halo are the debris left over from the accretion of dwarf galaxies. Unfortunately, it is impossible now to ascribe concrete nearest field RR Lyrae stars to some subsystem, because we do not know any intrinsic distinctive quality for them.

Some papers (Chiba \& Yoshii 1998; Martin \& Morrison 1998; Dambis \& Rastorguev 2001) present detailed studies of 
the kinematics of RR Lyrae stars in the solar neighborhood. The stars were assumed to form only one subsystem in a metalpoor halo. We use spatial velocities and computed elements of galactic orbits as our main criteria for isolating subsystems (because of the local, near-solar position of the studied RR Lyrae stars). Our wish is to investigate relationships between physical, chemical, spatial and kinematical characteristics of RR Lyraes stars in each metal-poor subsystem, determine the characteristic parameters of these subsystems, and compare them with the parameters of similar subsystems of globular clusters.

\section{The data}

We used for this study the largest catalogue of RR Lyrae variables, compiled by Dambis \& Rastorguev (2001). The catalogue contains 262 stars with published photoelectric photometry, metallicities, radial velocities, and absolute proper motions. Dambis \& Rastorguev (2001) used the proper motions from Hipparcos, PPM, NPMI, and Four-Million Star Catalog (Volchkov et al. 1992); the proper motions from the last three ground-based catalogs have been reduced to the Hipparcos system. The metallicities were used mainly from Layden (1994) and Layden et al. (1996), whose metallicity scale is in conformity with metallicity scale of globular clusters (Zinn \& West 1984). Dambis \& Rastorguev (2001) used radial velocities mainly from the paper of Fernley et al. (1998), and Solano et al. (1997), and main magnitude $\langle V\rangle-$ mainly from Fernley et al. (1998). Bearing in mind the large relative errors of trigonometric parallaxes for distant objects, we chose the photometric distance scale of Dambis \& Rastorguev (2001), assuming $M_{\mathrm{v}}(R R)=0.26[\mathrm{Fe} / \mathrm{H}]+1.17$. For each star, we computed the spatial velocity components in the cylindrical coordinates and the orbital elements using the Galaxy model from Allen \& Santillan (1991), which includes a spherical bulge, a disk, and an extended massive halo. The model assumes the galactocentric distance of the Sun to be $R_{\odot}=8.5 \mathrm{kpc}$ and the Galactic circular rotation velocity at the solar distance to be $V_{\text {rot }}=220 \mathrm{~km} \mathrm{~s}^{-1}$. The orbital elements were computed by modeling five complete revolutions around the galactic center for each star. The most informative quantities are: $Z_{\max }-$ the maximum height of the star above the galactic plane, $R_{\mathrm{a}}-$ the orbital apogalactic radius; $R_{\mathrm{p}}$ - the orbital perigalactic radius, and the eccentricity, $e=\left(R_{\mathrm{a}}-R_{\mathrm{p}}\right) /\left(R_{\mathrm{a}}+R_{\mathrm{p}}\right)$. A choice of stars based solely on their variability type and their visible magnitude ensures an absence of kinematical selection effects in the catalogue used. Our final sample of the metal-poor RR Lyrae variables contains 209 stars with metallicity $[\mathrm{Fe} / \mathrm{H}]<-1.0$. This simple metallicity criterion eliminates objects that belong to the thick disk subsystem of the Galaxy (see substantiations of Borkova \& Marsakov 2002).

\section{Criteria to select the subsystems}

It is much more difficult to identify objects that have an extragalactic origin, i.e. those belonging to the accreted halo. According to the hypothesis that the protogalaxy collapsed monotonically from the halo to the disk, suggested by
Eggen et al. (1962), stars genetically related to the Galaxy cannot have retrograde orbits. Only the oldest halo stars may be an exception, since they could have retrograde orbits due to the natural initial velocity dispersion of protostellar clouds. On the other hand, some stars formed from extragalactic fragments and captured by the Galaxy may have a prograde orbits. In any case, such stars should have fairly large peculiar spatial velocity relative to the local standard of rest, $V_{\text {pec }}$. Figure 1a displays the relation between the peculiar velocity (assuming $\left.\left(U_{\mathrm{o}}, V_{\mathrm{o}}, W_{\mathrm{o}}\right)=(-10,10,6) \mathrm{km} \mathrm{s}^{-1}\right)$ and the azimuth (circular) velocity component, $\Theta$, for the RR Lyrae stars of our sample. The diagram shows that there is a transition from prograde to retrograde orbits around the galactic center near $V_{\text {pec }} \approx 280 \mathrm{~km} \mathrm{~s}^{-1}$. We also observe an abrupt increase of the dispersion of the circular velocity component and a break of the dependence of circular velocity as a function of peculiar velocity at the same place (see the dispersion bars and the regression lines in the diagram). Figure $1 \mathrm{~b}$ displays a significant increase in the scatter of stars in $Z_{\max }$ when crossing the same threshold peculiar velocity. The abrupt change in the apogalactic radii of the stellar orbits is even more evident (Fig. 1c). First, the mean value of Ra remains much the same and when peculiar velocity crosses the threshold level, the mean value of apogalactic distances monotonically increase and their scatter sharply extends. We can see in Fig. 1d that the perigalactic distances also demonstrate the break of their relations in the vicinity of the same threshold peculiar velocity. The orbital eccentricities (Fig. 1e) not only abruptly increase their dispersion when crossing the same point, they also demonstrate different relations. First, the orbital eccentricities increase almost linearly with the peculiar velocities, reaching a maximum near the threshold velocity level. With further increase of $V_{\text {pec }}$, the mean and the scatter of the eccentricities do not change, within errors. Figure 1f shows that RR Lyrae stars with velocities near the threshold level might have any orbital inclination up to orthogonal to the galactic plane, whereas the range of "permitted" inclinations continuously becomes narrower when velocity moves away from this threshold level on both sides. For these reasons, we adopt $V_{\mathrm{pec}}>280 \mathrm{~km} \mathrm{~s}^{-1}$ as the critical value for distinguishing stars of the outer accreted halo. Here we suppose that the stars with lower peculiar velocities have a galactic origin, and belong to the proto-disk halo subsystem. Apparently, this kinematical criterion is not entirely unabbiguous: some stars of the proto-disk halo may have larger residual velocities. Evidence for this is provided, in particular, by the increase in the stellar density immediately to the right of the thereshold velocity level in our diagrams. However, we decided to retain a simple criterion, in order not to artificially confuse the situation.

Note again that the principal criterion distinguishing globular clusters of the accreted halo is their redder horizontal branches compared to clusters of the proto-disk halo. Moreover, empirical evidence suggests that the morphological structure of the horizontal branches is related to the mean period of RR Lyrae variables in metal-poor clusters (vanAlbada \& Baker 1972). We investigate the detailed data for RR Lyrae in globular clusters from Clement et al. (2001), and show that the redder the horizontal branch, the lower the mean period 


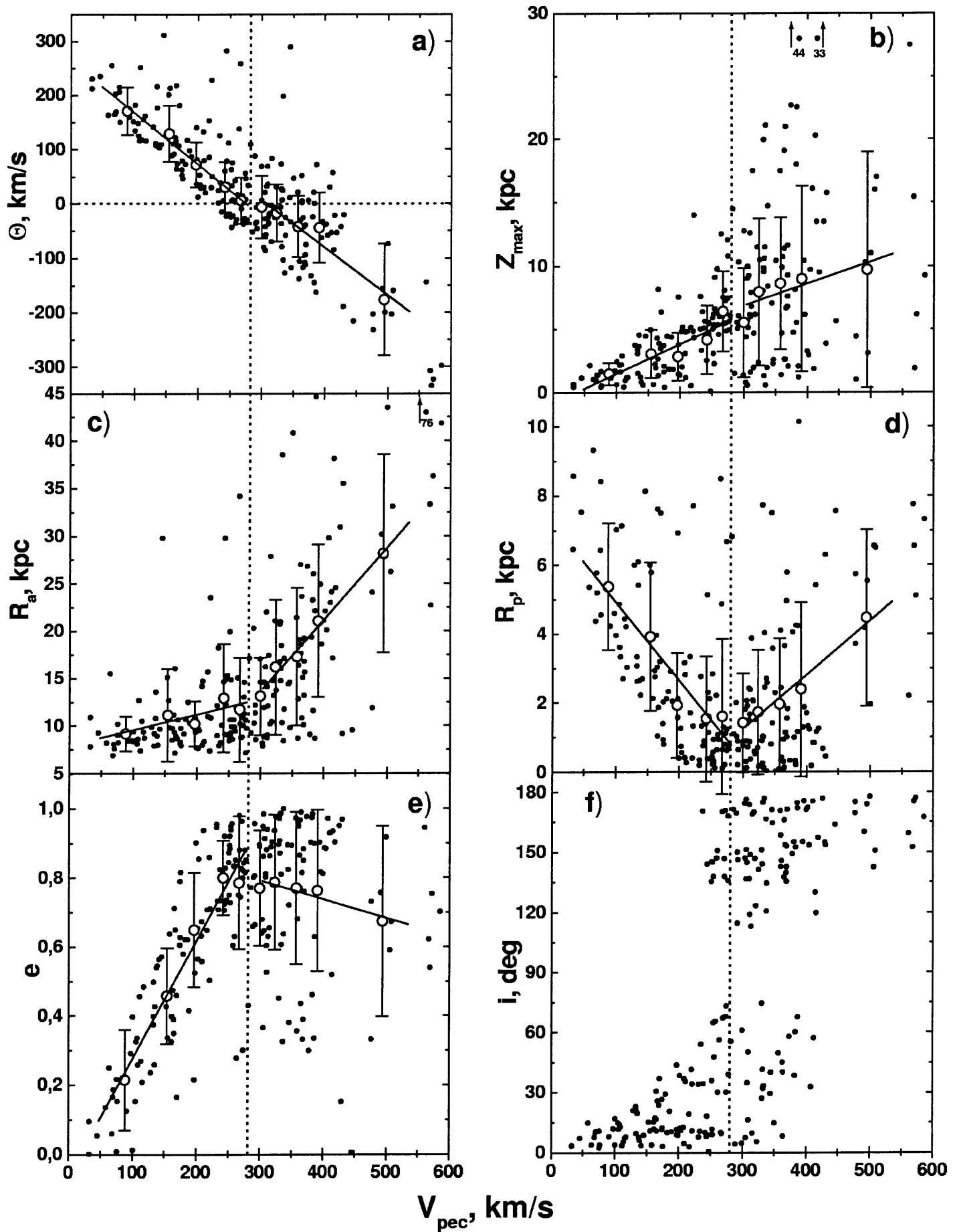

Fig. 1. The relations between the peculiar velocity relative to the local standard of rest, $V_{\text {pec }}$, and other characteristics of the RR Lyrae variables. Large open circles with error bars are mean values and dispersions of the corresponding parameters in narrow intervals $V_{\text {pec }}$. The straight lines are the least-square fits for the stars lying both to the left and on the right of $V_{\mathrm{pec}} \approx 280 \mathrm{~km} \mathrm{~s}^{-1}$ (the vertical dotted lines). An abrupt change in the relations and the dispersions near this threshold level is apparent in all panels.

with a height correlation coefficient $r=0.8 \pm 0.1$. It means that the mean period for RR Lyrae in clusters can be used to divide this cluster into subsystems. Therefore, it is interesting to test whether the variability period of single field RR Lyrae star may be adopted as well. To test this for field stars, we computed the mean periods of stars in a narrow metallicity range $(-1.7<$ $[\mathrm{Fe} / \mathrm{H}]<-1.2)$ for both halo subsystems. Each sample contained about sixty stars. It turns out that their mean periods are the same. Thus, the variability period of the field RR Lyrae stars cannot serve as an additional (internal) criterion to divide them into different subsystems of the metal-poor halo.

\section{Properties of stars in the subsystems}

Let us now compare the properties of the resulting subsystems. The spatial velocities of the stars can be used to obtain 
Table 1. Characteristics of metal-poor subsystems of field RR Lyrae variables and Globular Clusters.

\begin{tabular}{lcccc}
\hline \hline \multirow{2}{*}{ Characteristics } & \multicolumn{2}{c}{ Proto-disk halo } & \multicolumn{2}{c}{ Accreted halo } \\
\cline { 2 - 5 } & RR Lyr & GC & RR Lyr & GC \\
\hline$<[\mathrm{Fe} / \mathrm{H}]>, \mathrm{dex}$ & $-1.56 \pm 0.04$ & $-1.71 \pm 0.05$ & $-1.62 \pm 0.03$ & $-1.60 \pm 0.07$ \\
$\sigma_{[\mathrm{Fe} / \mathrm{H}]}, \mathrm{dex}$ & $0.33 \pm 0.05$ & $0.26 \pm 0.03$ & $0.32 \pm 0.02$ & $0.35 \pm 0.05$ \\
$<\Theta>, \mathrm{km} \mathrm{s}^{-1}$ & $93 \pm 8$ & $77 \pm 33$ & $-51 \pm 11$ & $-23 \pm 54$ \\
$\sigma_{\Theta}, \mathrm{km} \mathrm{s}^{-1}$ & $83 \pm 6$ & $129 \pm 19$ & $106 \pm 8$ & $140 \pm 18$ \\
$<e>$ & $0.57 \pm 0.03$ & $0.53 \pm 0.06$ & $0.76 \pm 0.02$ & $0.59 \pm 0.06$ \\
$\lim R_{\mathrm{a}}, \mathrm{kpc}$ & 18 & 10 & 42 & 20 \\
$\lim Z_{\mathrm{max}}, \mathrm{kpc}$ & 9 & 10 & 22 & 20 \\
$Z_{0}, \mathrm{kpc}$ & $2.6 \pm 0.2 *$ & $2.5 \pm 0.5$ & $6.1 \pm 0.3$ & $8.5 \pm 1.5$ \\
$\operatorname{grad}_{\mathrm{R}}[\mathrm{Fe} / \mathrm{H}], \mathrm{kpc}^{-1}$ & $-0.00 \pm 0.01$ & $-0.03 \pm 0.02$ & $-0.00 \pm 0.01$ & $-0.03 \pm 0.01$ \\
$\operatorname{grad}_{\mathrm{Z}}[\mathrm{Fe} / \mathrm{H}], \mathrm{kpc}^{-1}$ & $-0.02 \pm 0.01$ & $-0.03 \pm 0.02$ & $0.00 \pm 0.01$ & $-0.03 \pm 0.01$ \\
\hline
\end{tabular}

Note.* - The gradient of the proto-disk halo is strongly distorted by the selection which depends on only nearest stars.

an estimate for a number of characteristics of subsystems, if we can first reconstruct the stars' galactic orbits. Figure 2 shows the distributions of RR Lyrae in the two metal-poor subsystems as a function of their orbital elements. The top panels present the histograms of the rotation velocities for the sample of stars in the proto-disk halo and in the accreted halo. It is worth nothing that the two distributions can be properly fitted by a Gaussian (see the solid lines). Their maxima are separated by almost the dispersion (Table 1). The second row of graphs in Fig. 2 shows the corresponding distributions in orbital eccentricities. Here the characters of the histograms are obviously very different. All eccentricities are present in approximately equal number in the proto-disk halo (only a small excess is seen towards the high eccentricity side). Stars with very eccentric orbits prevail in the outer halo (where almost two thirds of all stars have $(e>0.8)$ ). The next row (Figs. 2e,f) presents distributions of orbital inclinations. The stars of both subsystems can have any orbital inclination. In both cases, the number of stars strongly increases with decreasing inclination; however, this is true for stars with prograde orbits in the proto-disk halo, and for those with retrograde orbits in the outer halo. We must bear in mind that the deficiency of stars with large orbital inclinations is largely due to the kinematical selection effect imposed on the sample of nearby stars. The vertical components of the spatial velocities of such stars in the solar neighborhood should be comparable to the galactic rotational velocity at this distance. Thus, the probability of their presence here is very low.

The fourth row of the histograms (Figs. $2 \mathrm{~g}, \mathrm{~h}$ ) can be used to estimate radial sizes of the subsystems and the fifth row (Figs. 2i,j), their vertical sizes. To quantitatively estimate the outer sizes of the subsystems based on these distributions, we reject the five most distant points in each histogram. Therefore, we simultaneously remove the largest uncertainties in the determination of the orbital elements and avoid possible errors in assigning some stars in our sample to a particular subsystem. Such estimates indicate that the size of the outer halo is approximately a factor of two larger than that one of the proto-disk halo (Table 1). The sizes of the subsystems in the direction perpendicular to the galactic plane also differ drastically (Table 1).

It is obviously not correct to compute the scale height using $Z_{\max }$, since all stars of a subsystem cannot simultaneously be located at the the highest points of their orbits. To reconstruct the "real", instantaneous Z-distribution for all stars, we must "spread" each star over its orbit from $-Z_{\max }$ to $+Z_{\max }$ in proportional to the probability density of its location at different $Z$. (This operation also smoothes fluctuations in the histogram due to the limited number of stars in the sample.) This probability density can easily be found from the computed orbit of the stars. The details of the procedure were written by Marsakov \& Shevelev (1995). The filled dots in the histograms of the bottom row (Figs. 2i,j) are the final reconstructed distributions in $Z$. In other words, this is how the stars will be distributed in height after some time, if they are rundomly distributed in their orbits. The solid curves in Figs. 3i,j are least-square approximations of the reconstructed distributions using an exponential law,

$n=\alpha \mathrm{e}^{-Z / Z_{0}}$,

where $Z_{0}$ is the scale height (the corresponding $Z_{0}$ values are indicated in Figs. 3i,j and in Table 1).

Let us now consider the metallicity gradients in the subsystems. Figure 3 displays the $R_{\mathrm{a}}-[\mathrm{Fe} / \mathrm{H}]$ and $Z_{\max }-[\mathrm{Fe} / \mathrm{H}]$ diagrams. The straight lines are the least-square regressions. Assuming that the stars are born near the apogalactic radii of their orbits, the slopes of these lines reflect the initial radial and vertical metallicity gradients for the subsystems. To increase the accuracy of the gradient estimates, we rejected the most distant data points in each case (see the vertical dotted lines in the diagrams). The resulting gradients are presented in Table 1 and the correlation coefficients in the corresponding diagrams. Only the vertical gradient in the proto-disk halo exceeds the error, but the correlation coefficient given in Fig. 3c tends to zero if we add the rejected distant stars. Note that RR Lyrae metallicities from Zinn \& West (1984) are estimated using the $\Delta S$ method, which actually measures calcium abundance. According to current evidence there is a systematic uncertainty between the metallicity scale suggested by Zinn \& West (1984) and other ones (see Rutledge et al. 1997; Rey et al. 2000). Therefore, to check our result we have to use more reliable metallicity determinations for field RR Lyrae stars, when sufficient data will be available. Thus the existence of vertical gradient in the proto-disk halo is still an open question. In contrast, according to our investigation, the complete absence 
Proto-disk halo

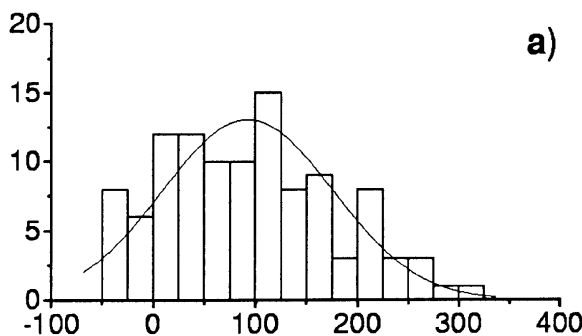

c)
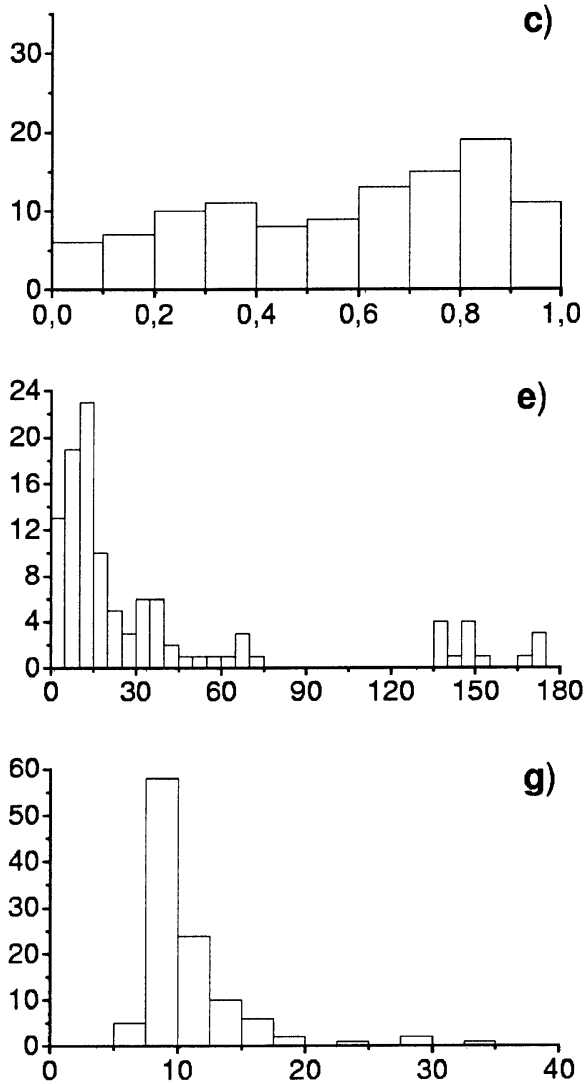

g)



\section{Accreted halo}

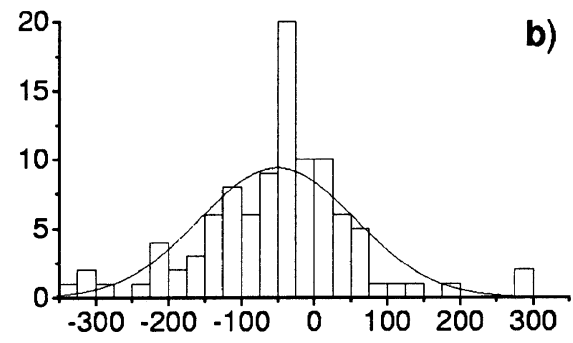

d)
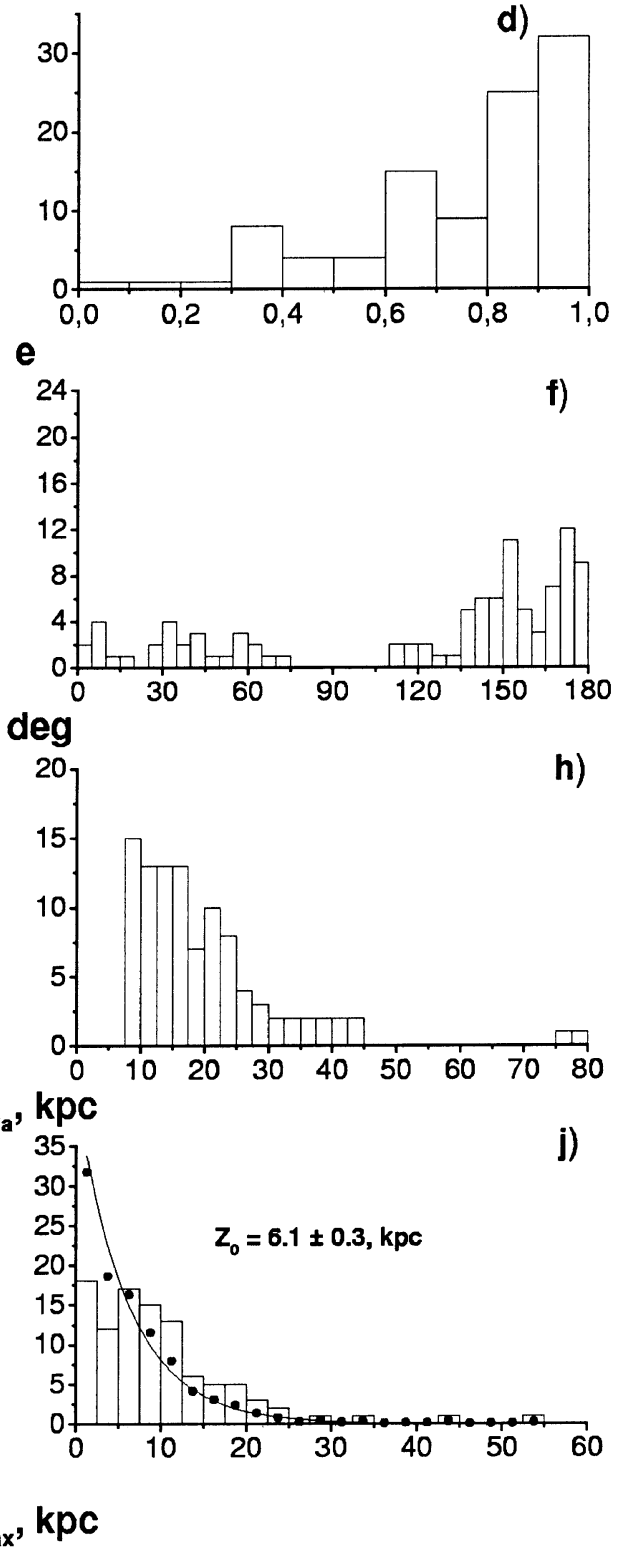

Fig. 2. Distributions of orbital elements for the RR Lyrae stars. Result for stars with $V_{\text {pec }}<280 \mathrm{~km} \mathrm{~s}^{-1}$ (left column), $V_{\text {pec }}>280$ (right colimn). The curves in the top row represent an approximation of the distributions of Gaussian functions. In bottom row, the dots are the reconstructed distributions of RR Lyrae stars in $Z$ (see the text for details), and the curves approximate the reconstructed distributions with an exponential law (the corresponding scale heights and their uncertainties are indicated).

of a radial gradient in the proto-disk halo and of both gradients in the accreted halo for the RR Lyrae stars is beyond doubt.

Suntzeff et al. (1991) investigate radial and vertical metallicity gradients for the metal-poor field RR Lyrae population as a whole. They used the present positions of stars in selected regions of the Galaxy in the galactocentric distance range 4-30 kpc. The authors indicate that outside the solar circle the metallicity gradients are zero as a function of $R_{\mathrm{a}}$ or $|Z|$. 
Proto-disk halo

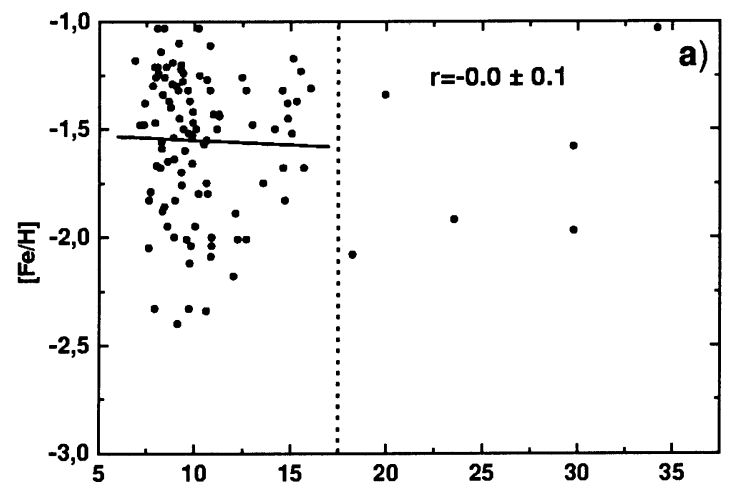

Accreted halo

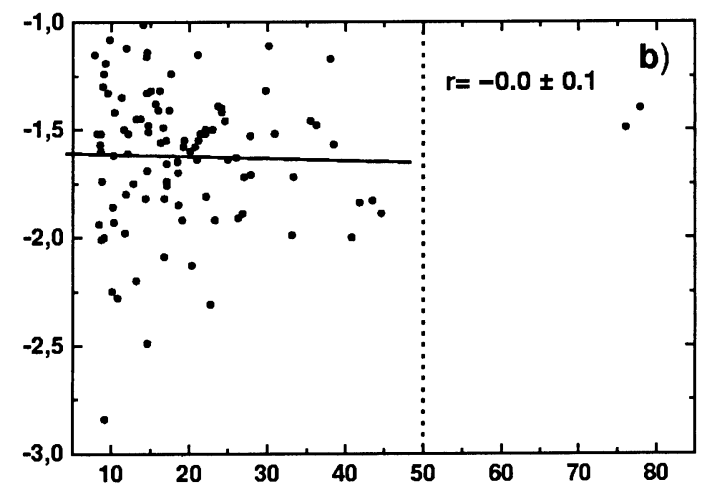

$R_{\mathrm{a}}, \mathrm{kpc}$



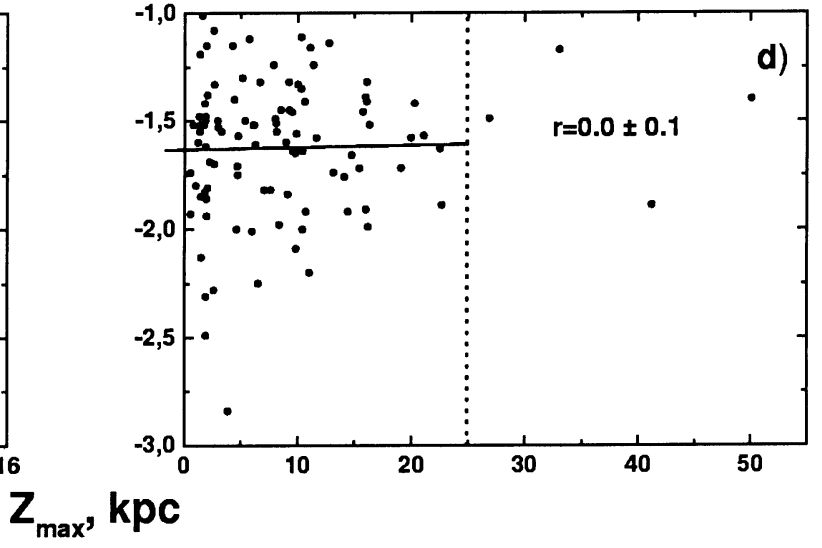

Fig. 3. Metallicities of the RR Lyrae stars versus apogalactic distance (a), b)) and maximum height above the galactic plane (c), d)). The straight lines are least-square fits. The slopes of the lines determine the metallicity gradients. The most distant stars in each panel (on the right of the dotted lines) were rejected from our computations. The correlation coefficient and their uncertainties are given.

This result is in agreement with gradients in our accreted halo estimated on $R_{\mathrm{a}}$ and $Z_{\max }$. Inside the solar circle they find the radial gradient of $-0.06 \pm 0.02 \mathrm{dex} \mathrm{kpc}^{-1}$, which is steeper than that of our proto-disk halo for field RR Lyraes (but is in agreement for globular clusters). This discrepancy is due to the lack of observed RR Lyraes inside the solar circle. The vertical gradient in $[\mathrm{Fe} / \mathrm{H}]$ they estimate as roughly $-0.05 \mathrm{dex} \mathrm{kpc}^{-1}$ out to $3 \mathrm{kpc}$. Our value for the proto-disk halo is larger because Suntzeff et al. (1991) did not divide the metal-poor stars into subsystems. So we may conclude that computed orbital elements for the nearest stars are good enough for estimation of metallicity gradients in the much larger distance range (but not inside the solar circle.)

\section{Discussion and conclusion}

Here, we will compare the characteristics of the metal-poor RR Lyrae subsystems derived in this study to the parameters of corresponding subsystems of globular clusters from Borkova \& Marsakov (2000), since only globular clusters were distinguished according to an intrinsic, physical parameter rather than interrelated spatial and kinematical criteria. The parameters of the metallicity distributions of the corresponding halo subsystems differ somewhat. In particular, the mean metallicity of the proto-disk halo derived from the globular clusters is lower than that of the outer halo. The metallicity dispersion is also lower. The field stars show the opposite pattern (Table 1). In all cases, however, the differences are comparable to the formally computed uncertainties, indicating that any conclusions about differences between these parameters have low statistical significance. The vertical gradients in the proto-disk halo for both the RR Lyrae stars and the globular clusters almost coincide, but the radial gradients differ (see Table 1). In the accreted halo, both gradients are absent for the field RR Lyrae stars but are non-zero for the clusters. However, both gradients for the globular clusters in the accreted halo are due exclusively to metal-richer objects close to the galactic center $(R \sim 7 \mathrm{kpc})$. Distant RR Lyrae were not included in our sample. In any case, values of all corresponding gradients coincide within the uncertainties. In this study, we have identified the halo subsystems based on spatial velocity. Therefore, the differences between the proto-disk halo and the accreted halo in any kinematical parameter for field stars should be more prominent. Indeed, while the difference between the orbital velocities for the globular clusters of the proto-disk halo and of the accreted halo is $\sim 100 \mathrm{~km} \mathrm{~s}^{-1}$ this difference is approximately $40 \%$ higher for the RR Lyrae variables (see Table 1). The velocity dispersions for the subsystems of globular clusters are obviously overestimated due to the large distance uncertainties and, as a result, are much higher than the values for field RR Lyrae stars. 
The mean eccentricities in the proto-disk halo subsystems are the same, whereas in the outer halo the eccentricities are, on average, higher for the field stars, as expected (note that the proper motions and, hence, orbital eccentricities are known only for a small number of clusters, and with large uncertainties). The radial size of the proto-disk halo is approximately a factor of 1.8 larger for the field stars than for the clusters, whereas the two scale heights were the same within the errors. Recall that we can estimate the radial sizes of RR Lyrae subsystems only from their maximum distances from the galactic center, which leads to appreciable overestimates of these sizes. The radial and vertical sizes of the outer accreted halo subsystem of field stars are naturally the largest, and are in reasonable agreement with the corresponding sizes for the subsystem of globular clusters. Note that, in order to obtain correct estimates of sizes of galactic subsystems based on data for nearby stars, it is necessary to take into account the kinematical selection effect, which leads to a deficiency of stars with large $R_{\mathrm{a}}$ and $Z_{\max }$ in the solar neighborhood.

Thus, we found that the generally good agreement between the characteristics of corresponding subsystems of field RR Lyrae stars and globular clusters, distinguished using different criteria, shows that both populations are not uniform. Both the clusters and field stars belong to two spherical subsystems of the Galaxy: the inner proto-disk halo (related to the disk by its origin) and the outer accreted halo. The collected results indicate that this subsystem is characterized by large size, an absence of appreciable metallicity gradients, predominantly large orbital eccentricities, a large number of objects on retrograde orbits, and, on average, younger ages for its objects, supporting the hypothesis that objects in this subsystem have an extragalactic origin.
Acknowledgements. This study was supported by the RFBR (projects 00-02-17689 and 02-02-06911).

\section{References}

Allen, C., \& Santillan, A. 1991, Rev. Mex. Astron. Astrofis., 22, 255

Borkova, T. V., \& Marsakov, V. A. 2000, Astron. Rep., 44, 665

Borkova, T. V., \& Marsakov, V. A. 2002, Astron. Rep., 46, 460

Chiba, M., \& Yoshii, Y. 1998, AJ, 115, 168

Clement, C. M., Muzzin, A., Dufton, Q., et al. 2001, AJ, 122, 2587

Da Costa, G. S., \& Armandroff, T. E. 1995, AJ, 109, 2533

Dambis, A. K., \& Rastorguev, A. S. 2001, AstL, 27, 108

Eggen, O. J., Linden-Bell, D., \& Sandadge, A. 1962, ApJ, 136, 748

Fernley, R., Burnes, T. G., Solano, I., et al. 1998, A\&A, 330, 515

Hartwick, F. D. A. 1987, in The Galaxy, ed. G. Gilmore, \& B. Carwell (Reidel, Dordrecht), 281

Ivezic, Z., Goldston, J. Fintlator, K., et al. 2000, AJ, 120, 963

Layden, A. C. 1994, AJ, 108, 1016

Layden, A. C., Hanson, R. B., Hawley, S. L., et al. 1996, AJ, 112, 2110

Marsakov, V. A., \& Shevelev, Yu. G. 1995, Astron. Rep., 39, 559

Martin, J. C., \& Morrison, H. L. 1998, AJ, 116, 172

Rey, S. C., Lee, Y. W., Joo, J. M., et al. 2000, AJ, 119, 1824

Rutledge, G. A., Hesser, J. E., \& Stetson, P. B. 1997, PASP, 109, 907

Solano, E., Garrido, R., Fernley, R., \& Burnes, T. G. 1997, A\&AS, 125,321

vanAlbada, T. S., \& Baker, N. 1972, ApJ, 169, 311

Volchkov, A. A., Kuzmin, A. V., \& Nesterov, V. V. 1992, in FourMillion Star Catalog, ed. A. P. Guliaev, \& V. V. Nesterov (MSU, Moscow), 67

Zinn, R., \& West, M. J. 1984, ApJS, 55, 45

Zinn, R. 1993, in The Globular Cluster - Galaxy Connection, ed. H. Smith, \& J. Brodee, ASP Conf. Ser., 48, 38 